\title{
Barriers to following dietary recommendations for type 2 diabetes in patients from UK African and Caribbean communities: a qualitative study
}

\author{
A.P. Moore ${ }^{1}$, C.A Rivas ${ }^{2}$, S. Harding ${ }^{1}$ and L.M Goff ${ }^{1}$ \\ ${ }^{1}$ King's College London, Stamford Street, London SE1 9 NH and \\ ${ }^{2}$ University College London, Woburn Square, London WC1H OAA.
}

This abstract was awarded the student prize.

The prevalence of type 2 diabetes (T2D) is up to 3 times higher in UK African and Caribbean (AfC) communities than the general UK population ${ }^{1}$. Lifestyle change is the cornerstone of T2D self-management advice, but cultural barriers may limit engagement in dietary change for AfC patients, ${ }^{2}$. The aim of this study was to explore barriers to adopting healthy eating behaviours in people with T2D from London AfC communities.

Qualitative methods were used to understand factors influencing health behaviour. Focus groups were held with AfC T2D patients and were stratified by gender and ethnicity. Groups, held in the community, faith venues and on university premises, lasted 2-hrs and were digitally recorded and transcribed. NVivo software was used to manage the data coding and analysis. A thematic analysis approach was used to analyse the data; $20 \%$ of transcripts were independently coded for data validation. Results were triangulated with members of the community and an external peer reviewer for verification of key themes.

Focus groups were held with 41 patients (68\% female; $58 \%$ African, $42 \%$ Caribbean; $89 \%$ first generation UK immigrants; mean age $62 \mathrm{yrs})$. Barriers to following dietary recommendations included socio-cultural and lifestyle factors (figure 1). Traditional foods, high in carbohydrate and fat, were seen as central to cultural identity yet often not included in dietary recommendations, reducing the salience of healthcare advice. A rejection of weight loss targets and an acceptance of larger body size meant that reducing consump-

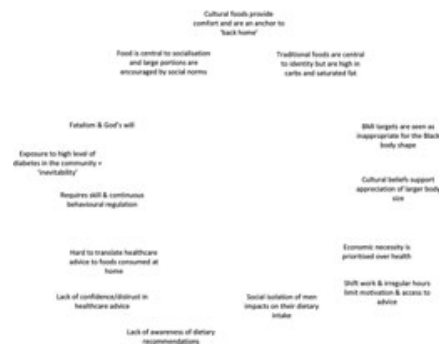

tion and awareness of calorie density of the diet was not a key focus. Dietary self-efficacy was reduced by a view of the inevitability of diabetes and the overwhelming task of continuous monitoring of consumption. Busy, stressful working lives limited motivation to engage in dietary management.

The salience of dietary advice may be improved if it is centred around culturally relevant foods. However, cultural beliefs and practical barriers also need to be considered to support healthful behaviour change in these communities.

1. Paul SK, Owusu Adjah ES et al. Diab. obes. \& met. (2017); 19:1014-23.

2. Abubakari AR, Jones MC et al. Int J Gen Med. (2013); 6:617-28.

3. Breen C, Ryan M et al. Brit. Journ. Nutrit. (2015); 114:439-47. 